Original Article

\title{
A comparison of trapezius muscle activities of different shoulder abduction angles and rotation conditions during prone horizontal abduction
}

\author{
Jin Yong Lim, MS, PT ${ }^{1)}$, Jung Seok Lee, MS, PT ${ }^{1)}$, Byeong Mu Mun, PhD, $\mathrm{PT}^{1}$, \\ TAE Ho KIM, PhD, $\mathrm{PT}^{1)^{*}}$ \\ 1) Department of Physical Therapy, College of Rehabilitation Science, Daegu University: 15 Jilyang, \\ Gyeongsan-si, Gyeongsangbuk-do 712-714, Republic of Korea
}

\begin{abstract}
Purpose] This study examined the differences in the activities of three parts of the trapezius muscle - the upper trapezius (UT), middle trapezius (MT), and lower trapezius (LT) - among three different rotation conditions of the shoulders, while subjects performed prone horizontal abduction (PHA) at $30^{\circ}, 60^{\circ}, 90^{\circ}$, and $120^{\circ}$ of abduction. [Subjects and Methods] The subjects of this experimental study were 16 healthy male adults. Surface electromyography was used to collect data on the activity of each part of the trapezius. A two-way analysis of variance was used to compare the activities of each area of the trapezius - the UT, MT, and LT - among internal rotation (IR), the neutral position (NP), and external rotation (ER) of the shoulders during PHA with shoulder abduction of $30^{\circ}, 60^{\circ}, 90^{\circ}$, and $120^{\circ}$. [Results] Activity of the UT, MT, and LT significantly increased as the shoulder abduction angle increased during PHA. There was a significant difference only in the activity of the LT, with change in shoulder rotation. In addition, the muscle activity of the LT was highest during shoulder IR at $120^{\circ}$. [Conclusion] Although activity of the LT was the highest during IR at 120 abduction, PHA accompanied by ER at an abduction angle of $120^{\circ}$ would be effective at eliciting high activity in the LT when PHA is performed. Nonetheless, at an early stage of rehabilitation, PHA accompanied by ER at low abduction angles of $30^{\circ}$ and $60^{\circ}$ would be desirable to elicit low activity of the UT and high activity of the LT.

Key words: Prone horizontal abduction, Trapezius muscle activity, Electromyography
\end{abstract}

(This article was submitted Jun. 12, 2014, and was accepted Jul. 18, 2014)

\section{INTRODUCTION}

The trapezius muscle, which adjusts the movement and location of the scapula, plays an important role in both the stability and movement of the shoulder joints and, therefore, is a crucial muscle for optimal upper extremity function ${ }^{1)}$. The trapezius changes the location of the glenoid fossa during shoulder abduction, which enables maintenance of the optimal position of the humeral head ${ }^{2}$. When the shoulder is abducted, the upper trapezius (UT), middle trapezius (MT), and lower trapezius (LT) become important muscles that adjust the upward rotation together with the serratus anterior muscle ${ }^{3)}$. In particular, the MT and LT greatly contribute to the maintenance of appropriate shoulder kinematics and stability.

Changes in the activity of the scapula muscle are discovered in patients with scapular dyskinesis ${ }^{4}$. When those with abducted scapular alignment, known as rounded shoulder, elevate their arm, their serratus anterior muscles are insuf-

*Corresponding author. Tae Ho Kim (E-mail: ptkimth@ daegu.ac.kr)

(C2015 The Society of Physical Therapy Science. Published by IPEC Inc. This is an open-access article distributed under the terms of the Creative Commons Attribution Non-Commercial No Derivatives (by-ncnd) License $<$ http://creativecommons.org/licenses/by-nc-nd/3.0/> ficiently activated. In addition, their MT and LT muscles have decreased activity in conjunction with excessive activity of the UT, which results in reduced upward rotation, external rotation (ER), and posterior tilt of the scapula ${ }^{5)}$.

Some studies have tested the activity of the scapular muscle through diverse exercises aimed at improving recruitment $^{6-8)}$. Recently, Cool et al. ${ }^{1)}$ selected four kinds of exercises for healthy subjects - anterior flexion in a side-lying position, ER in a side-lying position, horizontal abduction accompanied by ER in a prone position, and shoulder extension in a prone position with the arm in the neutral position (NP) - that focus on minimizing the activity of the excessively activated muscles and selectively activating the weakened muscles. Exercises aimed at increasing the ratio of MT and LT to UT have been proposed for improving scapulothoracic posture and decreasing imbalance in patients with shoulder pathology.

Many researchers have studied prone horizontal abduction (PHA) as an exercise for activating the MT and LT muscles ${ }^{9-11)}$. Moseley et al. ${ }^{9)}$ reported that one of the most optimal postures with high activity of both the MT and LT muscles was at $90^{\circ}$ PHA. Elissa et al. ${ }^{10)}$ measured the muscle activity of healthy subjects during PHA at shoulder abduction angles of $75^{\circ}, 90^{\circ}, 125^{\circ}$, and $160^{\circ}$ and reported that the MT and LT muscles were more greatly activated at $90^{\circ}$ and $125^{\circ}$ than at $160^{\circ}$. Although they studied the activ- 
ity of the trapezius muscle during PHA, research that has examined the differences in the activities of each part of the trapezius muscle according to shoulder joint rotation is lacking. Therefore, the purpose of this study was to investigate the muscle activity changes under shoulder rotation conditions of internal rotation (IR), NP, and ER at different abduction angles of $30^{\circ}, 60^{\circ}, 90^{\circ}$, and $120^{\circ}$ during PHA.

\section{SUBJECTS AND METHODS}

The subjects of this study were healthy adults residing in Daegu, and they voluntarily consented to participate in this experiment. The study period was from December 1, 2013 to March 1, 2014. The subjects were 16 healthy male adults who had no orthopedic or neurological problems, no injuries to the neck or head, and no history of surgical operation in the area (age: $28.93 \pm 4.18$ years old, weight: $67.12 \pm 3.91 \mathrm{~kg}$, height: $172.25 \pm 3.31 \mathrm{~cm}$, mean $\pm \mathrm{SD})$. The subjects provided their informed consent before participating in this study. The study was approved by the Institutional Review Board of Daegu University, in accordance with the ethical principles of the Declaration of Helsinki.

Surface electromyography (EMG) (Noraxon TeleMyo DTS wireless system, Noraxon Inc., AZ, USA) was used to measure the activity of each muscle. The activities of the muscles were collected and analyzed using Myoreserch XP 1.07 software on a PC. EMG signals were sampled at $1,000 \mathrm{~Hz}$, and filtered with a bandpass filter between 40 $250 \mathrm{~Hz}$ and a $60-\mathrm{Hz}$ notch filter.

With the subjects in an upright standing position, the surface electrode for the UT muscle was attached to the lateral middle area between the spinous process of the seventh cervical vertebra and the clavicle. The surface electrode for the MT muscle was attached to the skin between the middle area of the spine of the scapula and the spinous process at the same height, and the electrode for the LT muscle was attached about $5 \mathrm{~cm}$ below the root of the spine of the scapula ${ }^{12}$. Approximate locations of each muscle were marked with an oil-based pen. The muscle belly obviously seen when maximal muscle contraction was induced during manual muscle testing (MMT) was identified, and the areas for attaching the EMG electrodes were marked. The areas marked for attaching the electrodes were rubbed with thin sandpaper three to four times to remove the skin's horny layer, skin fat was removed with an alcohol swab, and the electrodes were attached with a distance between them of $2 \mathrm{~cm}^{13)}$.

For the experiment, the subjects lay on the treatment table in a prone position with their arm hanging down from the table at a flexion of $90^{\circ}$. Then, the four shoulder abduction postures were adopted: $30^{\circ}, 60^{\circ}, 90^{\circ}$, and $120^{\circ}$. PHA motion was performed for each shoulder rotation condition using only the weight of the arm, without resistance, in order to measure the muscle activities of the UT, MT, and LT muscles. ER was defined as the condition under which the thumb was uppermost, facing toward the ceiling. The neutral position (NP) was the condition when the thumb was facing the body, and internal rotation (IR) was the condition when the thumb was lowermost, facing toward the floor. With the elbow joint completely extended, the arm was raised to the trunk level. In order to help the subjects understand the experiment, preliminary exercises were conducted for five minutes using each angle and rotation. The abduction angle of the shoulders was set with a goniometer and each subject used their dominant arm. The orders of measurement were selected randomly from among the 4 abduction angles and 3 rotation positions of the shoulder joint. Randomization was conducted by an investigator using computer-generated random numbers.

For normalization of the EMG data obtained from the measurement, maximal voluntary isometric contraction (MVIC) of each muscle was measured three times, and a resting time of 30 seconds was provided between each measurement in order to reduce muscle fatigue. Five seconds of EMG data were converted to root mean square (RMS) values, and the value of the average EMG signal of the middle three seconds-excluding the first and last seconds-was converted to $\% \mathrm{MVIC}^{14)}$.

A two-way analysis of variance (ANOVA) was conducted in order to compare the muscle activities of each part of the trapezius muscle in the IR, NP, and ER conditions of the shoulder during PHA with shoulder abductions of $30^{\circ}, 60^{\circ}$, $90^{\circ}$, and $120^{\circ}$. The least significant difference test was used as a post hoc test. The significance level was chosen as 0.05 . The Statistical Package for Social Sciences (SPSS) version 12.0 was used for the statistical analysis.

\section{RESULTS}

Table 1 shows the average value of the muscle activity of each part of the trapezius muscle under each type of rotation at $30^{\circ}, 60^{\circ}, 90^{\circ}$, and $120^{\circ}$ of shoulder abduction. There was no significant interaction between angle and rotation in UT muscle activity $(p>0.05)$. There were significant main effects of each angle $(\mathrm{p}<0.05)$. According to the post-hoc test, there were significant differences between $30^{\circ}$ and $90^{\circ}$, $30^{\circ}$ and $120^{\circ}, 60^{\circ}$ and $90^{\circ}, 60^{\circ}$ and $120^{\circ}$, and $90^{\circ}$ and $120^{\circ}$ $(\mathrm{p}<0.05)$. There was significant interaction between rotation and angle in MT muscle activity $(p<0.05)$. There were significant main effects of each angle $(p<0.05)$. There were significant differences between $30^{\circ}$ and $60^{\circ}, 30^{\circ}$ and $90^{\circ}$, $30^{\circ}$ and $120^{\circ}, 60^{\circ}$ and $90^{\circ}, 60^{\circ}$ and $120^{\circ}$, and $90^{\circ}$ and $120^{\circ}$, according to the post-hoc test results $(\mathrm{p}<0.05)$.

There was significant interaction between rotation and angle in LT muscle activity $(\mathrm{p}<0.05)$. There were significant main effects of each angle and rotation $(p<0.05)$. There were significant differences between $30^{\circ}$ and $90^{\circ}, 30^{\circ}$ and $120^{\circ}, 60^{\circ}$ and $90^{\circ}, 60^{\circ}$ and $120^{\circ}$, and $90^{\circ}$ and $120^{\circ}$, according to the post-hoc test results $(\mathrm{p}<0.05)$. There were significant differences between ER and NP, and between IR and ER $(\mathrm{p}<0.05)$. The LT muscle showed the highest activity in IR at $120^{\circ}$ (Table 1$)$

\section{DISCUSSION}

Among the many exercises aimed at improving the recruitment of the muscles around the scapula, PHA is an exercise which activates the MT and LT muscles, and many researchers have studied it ${ }^{10,11)}$.

According to our present results, there was no significant 
Table 1. Activity of each muscle at each abduction angle

(Unit: \%MVIC)

\begin{tabular}{|c|c|c|c|c|c|}
\hline Muscle & Degree & IR & NP & ER & \\
\hline \multirow{4}{*}{ UT } & $30^{\circ}$ & $3.75 \pm 2.23$ & $4.31 \pm 2.15$ & $5.25 \pm 2.79$ & \multirow{4}{*}{ \#* } \\
\hline & $60^{\circ}$ & $5.69 \pm 2.18$ & $6.25 \pm 2.40$ & $7.31 \pm 3.17$ & \\
\hline & $90^{\circ}$ & $12.69 \pm 4.23$ & $15.25 \pm 6.03$ & $15.63 \pm 7.08$ & \\
\hline & $120^{\circ}$ & $29.38 \pm 11.93$ & $22.81 \pm 9.77$ & $23.25 \pm 10.69$ & \\
\hline \multirow{4}{*}{ MT } & $30^{\circ}$ & $9.31 \pm 4.28$ & $11.50 \pm 3.84$ & $16.44 \pm 5.60$ & \multirow{4}{*}{$\# * \S^{*}$} \\
\hline & $60^{\circ}$ & $16.69 \pm 6.51$ & $17.19 \pm 4.99$ & $21.13 \pm 5.85$ & \\
\hline & $90^{\circ}$ & $30.44 \pm 9.91$ & $36.75 \pm 14.46$ & $38.88 \pm 12.10$ & \\
\hline & $120^{\circ}$ & $52.13 \pm 15.51$ & $41.44 \pm 11.92$ & $41.06 \pm 9.28$ & \\
\hline \multirow{4}{*}{ LT } & $30^{\circ}$ & $10.31 \pm 7.31$ & $13.50 \pm 6.84$ & $26.81 \pm 6.69$ & \multirow{4}{*}{$¥^{*} \#^{*} \S^{*}$} \\
\hline & $60^{\circ}$ & $13.50 \pm 6.17$ & $17.44 \pm 8.99$ & $31.44 \pm 10.18$ & \\
\hline & $90^{\circ}$ & $28.88 \pm 10.91$ & $35.81 \pm 12.72$ & $45.56 \pm 13.45$ & \\
\hline & $120^{\circ}$ & $61.88 \pm 11.58$ & $51.75 \pm 12.10$ & $56.88 \pm 12.20$ & \\
\hline \multicolumn{6}{|c|}{$\begin{array}{l}\text { UT: upper trapezius, MT: middle trapezius, LT: lower trapezius, IR: internal rotation, NP: neu- } \\
\text { tral position, ER: external rotation } \\
\text { ¥ Indicates a significant main effect of rotation, \# Indicates a significant main effect of abduction } \\
\text { angle, § Indicates a significant interaction between rotation and abduction angle }\end{array}$} \\
\hline
\end{tabular}

difference in the muscle activities of the UT and MT between any of the rotation conditions. However, the muscle activities significantly increased as the angle increased. There was a significant increase in the activity of the LT muscle with both changes in angle and rotation. This result signifies that the activities of the UT and MT are not affected by the rotation condition of the shoulders, only the LT muscle being affected by the rotation condition during PHA. In addition, the activity of each part of the trapezius muscle significantly increased as the angle increased. We consider that the activities of the three parts of the trapezius muscle increased with the rise in abduction angle because, as the abduction angle increases, upward rotation and posterior tilt of the scapula also increase. Another influential factor is the difference in the length of the humeral lever arm as the abduction angle of the shoulders increases. Further, the activity of the MT and LT increased until the abduction angle became $120^{\circ}$, a result similar to the results of a study by Ekstrom et $\mathrm{al}^{15)}$. In that study, the activities of the MT and LT were highest in exercises raising the arms over the head along a line in the direction of the LT muscle fibers. In a study of PHA by Elissa et al. ${ }^{10)}$, activity of the MT and LT was high at $90^{\circ}$ and $125^{\circ}$ during PHA, a result which is supported by our present study results.

Only activity of the LT changed with the rotation condition. The activity of the LT increased in the order of IR, NP, and ER at $30^{\circ}, 60^{\circ}$, and $90^{\circ}$, respectively, but it was highest in IR and lowest in NP at $120^{\circ}$. At $30^{\circ}, 60^{\circ}$, and $90^{\circ}$, the activity of the LT was higher during PHA with ER than during PHA with IR. The reason for this result is that PHA with ER triggers accessory ER, posterior tilt, and depression of the scapula ${ }^{16)}$. Such motions are caused by activity of the LT. However, what is noteworthy in the present study is that the activity of the LT was higher during PHA accompanied by IR with abduction at $120^{\circ}$ than during ER. This is because PHA accompanied by IR triggers reduction in the scapular space in a $120^{\circ}$ abduction posture, causing compensatory elevation of the scapula to sufficient space and, as a result, more activity of the $\mathrm{UT}^{17}$ ). Here, activity of the LT is considered to increase in order to counteract the UT, which brings about excessive elevation of the scapula. During the $120^{\circ}$ PHA exercise accompanied by IR, the activity of the UT becomes higher, although its activity is not significantly different from the UT activity under the conditions of NP and ER. In addition, even though such exercise triggers higher activity of the LT, it is not a single and voluntary activity of the LT but a secondary action, elicited by the activity of the UT. Therefore, it is not a selective exercise for the LT.

PHA is a trapezius muscle strengthening exercise which is widely used in clinical practice. Nonetheless, shoulder angle and rotation during PHA exercise have been little considered. The present study results demonstrate that arm abduction location and rotation are important during PHA which is used to activate the trapezius in clinical practice. When performing PHA in order to stabilize the shoulders and strengthen muscles, arm rotation angle and location should be emphasized as major factors which can be utilized to target different areas of the trapezius.

A limitation of this study is that kinematic data obtained from the scapulothoracic joint and the glenohumeral joint were not collected and, therefore, could not be quantified. Based on the result of this study of healthy people, future research should utilize motion analysis systems, as well as activity of each area of the trapezius muscle, to investigate the posture of patients with shoulder dysfunctions at different abduction angles and rotation conditions.

In conclusion, PHA accompanied by ER with shoulder abduction at $120^{\circ}$ should be effective at inducing high activity in the LT when PHA is performed by patients with scapular dyskinesis. Nevertheless, during the early stage of rehabilitation, PHA accompanied by ER at low angles of $30^{\circ}$ or $60^{\circ}$ is desirable to lower the activity of the UT and raise the activity of the LT. 


\section{REFERENCES}

1) Cools AM, Declercq GA, Cambier DC, et al.: Trapezius activity and intramuscular balance during isokinetic exercise in overhead athletes with impingement symptoms. Scand J Med Sci Sports, 2007, 17: 25-33. [Medline]

2) Mottram SL: Dynamic stability of the scapula. Man Ther, 1997, 2: 123 131. [Medline] [CrossRef]

3) Lehman GJ, Buchan DD, Lundy A, et al.: Variations in muscle activation levels during traditional latissimus dorsi weight training exercises: an experimental study. Dyn Med, 2004, 3: 4. [Medline] [CrossRef]

4) Yoo WG: Comparison of shoulder muscles activation for shoulder abduction between forward shoulder posture and asymptomatic persons. J Phys Ther Sci, 2013, 25: 815-816. [Medline] [CrossRef]

5) Ludewig PM, Braman JP: Shoulder impingement: biomechanical considerations in rehabilitation. Man Ther, 2011, 16: 33-39. [Medline] [CrossRef]

6) Johnson GR, Pandyan AD: The activity in the three regions of the trapezius under controlled loading conditions - an experimental and modelling study. Clin Biomech (Bristol, Avon), 2005, 20: 155-161. [Medline] [CrossRef]

7) Lee SK: The effects of vibration stimuli applied to the shoulder joint on the activity of the muscles around the shoulder joint. J Phys Ther Sci, 2013, 25: 1407-1409. [Medline] [CrossRef]

8) Reinold MM, Escamilla RF, Wilk KE: Current concepts in the scientific and clinical rationale behind exercises for glenohumeral and scapulothoracic musculature. J Orthop Sports Phys Ther, 2009, 39: 105-117. [Med- line] [CrossRef]

9) Moseley JB Jr, Jobe FW, Pink M, et al.: EMG analysis of the scapular muscles during a shoulder rehabilitation program. Am J Sports Med, 1992, 20: 128-134. [Medline] [CrossRef]

10) Kinney E, Wusthoff J, Zyck A, et al.: Activation of the trapezius muscle during varied forms of Kendall exercises. Phys Ther Sport, 2008, 9: 3-8. [Medline] [CrossRef]

11) Arlotta M, Lovasco G, McLean L: Selective recruitment of the lower fibers of the trapezius muscle. J Electromyogr Kinesiol, 2011, 21: 403-410. [Medline] [CrossRef]

12) Cools AM, Witvrouw EE, Declercq GA, et al.: Scapular muscle recruitment patterns: trapezius muscle latency with and without impingement symptoms. Am J Sports Med, 2003, 31: 542-549. [Medline]

13) Cram J, Kasman G, Holtz J: Introduction to surface electromyography. Gaithersburg: Aspen publishers, 1998, pp 81-89.

14) Kendall FP, McCreary EK, Provance PG, et al.: Muscles, testing and function: with posture and pain. Baltimore: Williams \& Wilkins, 1993, pp 329-331.

15) Ekstrom RA, Donatelli RA, Soderberg GL: Surface electromyographic analysis of exercises for the trapezius and serratus anterior muscles. J Orthop Sports Phys Ther, 2003, 33: 247-258. [Medline] [CrossRef]

16) Neumann DA: Kinesiology of the musculoskeletal system: foundations for rehabilitation. St. Louis: Mosby, 2013, pp 146-150.

17) Ludewig PM, Cook TM: Alterations in shoulder kinematics and associated muscle activity in people with symptoms of shoulder impingement. Phys Ther, 2000, 80: 276-291. [Medline] 